\title{
Sema4A Responds to Hypoxia and Is Involved in Breast Cancer Progression
}

\author{
Xiao Liu, ${ }^{a, b}$ Yinping Sun, ${ }^{b}$ Wei Tian, ${ }^{b}$ Fuli Wang, ${ }^{b}$ Xiao Lv, ${ }^{b}$ Min Wang, ${ }^{b}$ Tiantian Sun, ${ }^{b}$ \\ Jingjing Zhang, ${ }^{c}$ Lin Wang, ${ }^{*, d}$ and Mingyong Han ${ }^{*, a}$ \\ ${ }^{a}$ Cancer Therapy and Research Center, Shandong Provincial Hospital affiliated to Shandong University; Jinan \\ 250021, China: ${ }^{b}$ Zibo Central Hospital; Zibo 250000, China: ${ }^{c}$ Clinical Test Department of Shandong Qianfoshan \\ Hospital; Jinan 250014, China: and ${ }^{d}$ Research Center for Medicinal Biotechnology, Key Laboratory for Rare \& \\ Uncommon Diseases of Shandong Province, Shandong Academy of Medicinal Sciences; Jinan 250062, China. \\ Received June 4, 2018; accepted September 9, 2018; advance publication released online September 28, 2018
}

Semaphorin4A (Sema4A) is a family member of semaphorins expressed in immune cells and is also related with disease progression of tumor disease. In this study, we investigate the expression and pathological role of Sema4A in breast cancer (BCa). Our data showed that the expression of Sema4A increased in the tissues and serum of BCa patients when compared with normal controls. The expression of Sema4A in BCa cells could be induced by hypoxic treatment, whereas silencing hypoxia-inducible factor (HIF)-1 $\alpha$ could attenuate the above induced. Furthermore, chromatin immunoprecipitation (ChIP) analysis demonstrated that HIF-1 $\alpha$ could regulate the expression of Sema4A through directly binding to the promoter of Sema4A gene, whose enrichment could be further enhanced by hypoxic stimulation. In addition, silencing Sema4A could inhibit the proliferation, vascular endothelial growth factor (VEGF) production and the phosphorylation of Akt, extracellular signal-regulated kinase (ERK)1/2 mitogen-activated protein kinase (MAPK) and signal transduction and activator of transcription (STAT)3, but induce apoptosis of BCa cells in the presence of hypoxia. In contrast, recombinant human Sema4A treatment showed the opposite effects. Taken together, these results suggest that Sema4A could promote progression of $\mathrm{BCa}$ in the presence of hypoxia and it may hold potential for treatment target for $\mathrm{BCa}$.

Key words breast cancer; semaphorin; hypoxia; Semaphorin4A (Sema4A)

Semaphorins are originally identified to provide axon guidance cues for neural development. ${ }^{1)}$ Thus far, more than 30 Semaphorins have been found and they function through their receptors, called plexins. ${ }^{1)}$ In addition to its involvement in neural activity, Semaphorins are also related with the immune response and angiogenesis. ${ }^{2,3)}$ Recently, mounting evidence showed that Semaphorins contribute greatly to tumor progression and hold potential for treatment targets in various tumors. $^{3)}$

Semaphorin 4A (Sema4A) is a family member of Semaphorins and exists in a transmembrane form. Its extracellular form could be proteolytically cleaved and behave as soluble ligand in extra-cellular compartment. ${ }^{4)}$ As a pathological factor, it contributes to human diseases, such as viral bronchiolitis, ${ }^{5}$ asthma $^{5)}$ and retinal degenerative diseases ${ }^{6)}$ and exerts its effect on $\mathrm{T}$ cell ${ }^{7)}$ and macrophage. ${ }^{8)}$ Our previous study found that the elevated level of Sema4A in the synovial fibroblasts of rheumatoid arthritis could exacerbate its inflammatory phenotype. ${ }^{9)}$ Furthermore, soluble Sema4A could also promote the invasive ability of synovial fibroblasts. ${ }^{9)}$ In hepatocellular carcinoma, Sema4A promoted doxorubicin-induced epithelialmesenchymal transition (EMT) ${ }^{10)}$ Sun et al. also reported that Sema4A acted as a receptor for Plexin-B1 to induce migration of different tumor cells. ${ }^{11)}$

In this study, we aimed to characterize the expression and functional role of Sema4A in breast cancer $(\mathrm{BCa})$ cells and also investigate the pathological mechanism for the deregulated Sema4A in BCa.

\section{MATERIALS AND METHODS}

Collection of Human Tissue and Serum from Breast Cancer Patients Human breast tumor and its adjacent non-cancerous tissues were obtained from Qilu Hospital of Shandong University (Jinan, China). The serum samples were collected from Zibo Central Hospital (Zibo, China). This study protocol was approved by the institutional Review Board of Research Center for Medicinal Biotechnology of Shandong and the informed written consent was also obtained from the patients. Our work is also in accordance with The Code Ethics of the World Medical Association (Declaration of Helsinki).

Cell Culture and Small Interfering RNA (siRNA) Transfection MDA-MB-231 and MCF-7 were obtained from the American Type Culture Collection (ATCC, Manassas, VA, U.S.A.). Cells were cultured in Dulbecco's modified Eagle's medium (DMEM) or RPMI1640 supplemented with 10\% heat-inactivated fetal bovine serum (FBS) at $37^{\circ} \mathrm{C}$ in $5 \%$ $\mathrm{CO}_{2}$ atmosphere. For hypoxic treatment, cells were cultured in a 1 or $0.2 \% \mathrm{O}_{2}$ Anaerobic Glove Cabinet (Thermo Scientific) for $24 \mathrm{~h}$. For siRNA transfection, Sema4A siRNA (50 nm, SI00133560), hypoxia-inducible factor (HIF)-1 $\alpha$ (SI02664431), HIF-2 $\alpha$ (SI02663038) and Negative control (SI03650318) were bought from QIAGEN (Hilden, Germany) and transfected in to $\mathrm{BCa}$ cells by Hyperfect transfection reagent (QIAGEN). Recombinant human Sema4A protein was bought from Abnova.

Enzyme-Linked Immunosorbent Assay (ELISA) Sema4A in the patients' serum and culture media were detected as described before. ${ }^{9)}$ Vascular endothelial growth factor (VEGF) levels in culture supernatants were measured using ELISA 
A

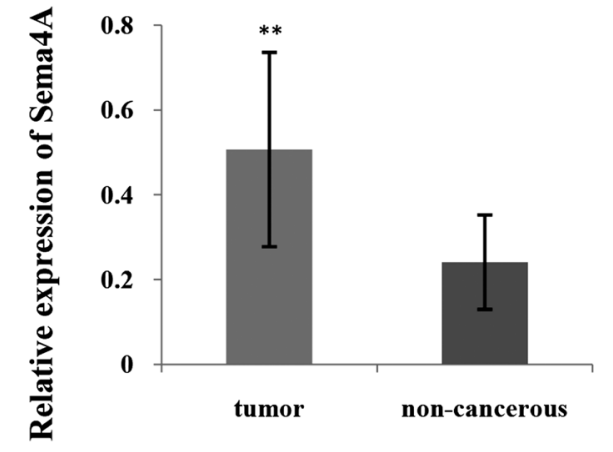

C

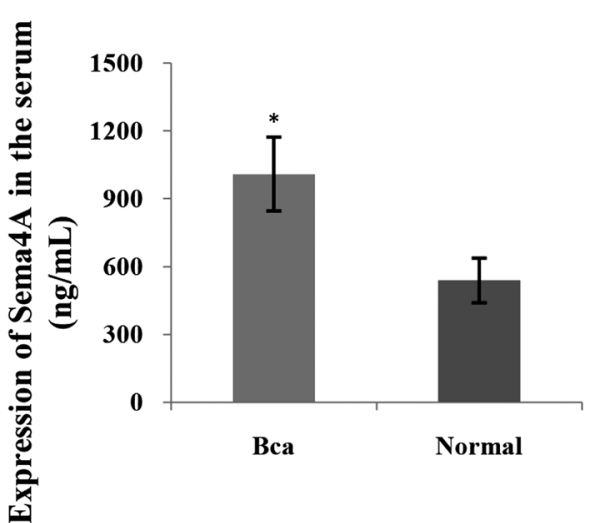

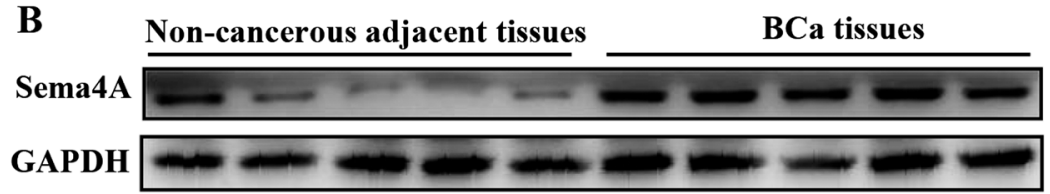

Fig. 1. Increased Sema4A Expression in $\mathrm{BCa}$

Expression of Sema4A at RNA (A) and protein (B) levels in BCa tissues $(n=5)$ and its non-cancerous adjacent tissues were detected by qPCR and Western blot. (C) The serum levels of Sema4A in BCa patients were determined by ELISA. $\left(* p<0.05,{ }^{*} p<0.05\right)$.

kits provided by R\&D Systems (Minneapolis, MN, U.S.A.).

RNA and RT Quantitative Real-Time PCR (qPCR) Total RNA was extracted from cells using Trizol reagent (Invitrogen) and reversely transcribed into cDNA by with a reverse transcription kit (TOYOBO, Osaka, Japan). Sema4A and VEGF were detected by qPCR using the following primer sequences: Sema4A forward: 5'-TAAAGTGAA TGA AAC CAT TTG T-3'; reverse: 5'-GTC TGT GAA ATG TTT TACA GTG T-3; glyceraldehyde-3-phosphate dehydrogenase (GAPDH) forward: 5'-CACCATCTTCCA GGA GC-3'; reverse: 5'-AGTGGACTCCACGACGTA-3'. The data shown were the relative changes as calculated by the $2-\Delta \Delta \mathrm{Ct}$ method and GAPDH was the internal control.

Western Blot Cells were lysed using lysis buffer (Beyotime, Shanghai, China) and the extracted protein was subjected to sodium dodecyl sulfate-polyacrylamide gel electrophoresis (SDS-PAGE), followed by transfer on polyvinylidene difluoride (PVDF) membrane. Then, membranes were blocked in $5 \%$ non-fat milk for $1 \mathrm{~h}$ at room temperature. After incubated with the primary antibody against Sema $4 \mathrm{~A}$ at $4^{\circ} \mathrm{C}$ overnight and HRP-conjugated anti-rabbit secondary antibody for $1 \mathrm{~h}$ at $37^{\circ} \mathrm{C}$, proteins were visualized by ECL kit (Merck Millipore). Phosphorylated (p)-Akt, Akt, p-extracellular signalregulated kinase (ERK)1/2 mitogen-activated protein kinase (MAPK), ERK1/2MAPK, signal transduction and activator of transcription (STAT)3 and pSTAT3 were purchased from Cell Signaling (Boston, U.S.A.). Sema4A (Abcam) or GAPDH (Santa Cruz). Bound antibodies were revealed with horseradish peroxidase-conjugated secondary antibodies (Santa Cruz).

Cell Proliferation and Apoptosis Assay After transfection with siRNA or hypoxic treatment, the proliferation of cells in 96-well plates was analyzed by CCK-8 Kit (Solabio). For apoptosis analysis, cells were stained for Annexin V (BestBio), counterstained with propidium iodide and measure by Flow Cytometry (BD, Accuri C6 Plus).

Chromatin Immunoprecipitation (ChIP)-qPCR Assay ChIP was performed according to the manufacturer's instruction (Millipore). Input DNA was applied to normalize the enrichment of HIF- $1 \alpha$ on the promoter of Sema4A gene and the specific binding was analyzed by qPCR using the primers as followings: forward: 5'-CTA TTC ACA GGC TTG ATC A3'; reverse: 5'-AAA ATT AGT TCC ACT CTT-3'. Anti-HIF-1 $\alpha$ and irrelevant antibody (immunoglobulin $\mathrm{G}(\mathrm{IgG})$ ) were from Abcam (Cambridge, MA, U.S.A.) and Millipore (Billerica, MA, U.S.A.), respectively.

Statistical Analysis All statistical analyses were carried out using SPSS 16.0 (New York, NY, U.S.A.). All the experiments were repeated for at least three repeats independently. Student's $t$-test was applied to evaluate the comparison of two independent groups. Data was expressed as mean \pm standard error of the mean (S.E.M.). $p<0.05$ was considered significant.

\section{RESULTS}

Sema4A Expression Increases in BCa To clarify the functional role of Sema4A in $\mathrm{BCa}$, we firstly analyzed its expression in $\mathrm{BCa}$ tissue as well as its adjacent non-cancerous tissues. The data showed that the expression of Sema4A at both RNA (Fig. 1A) and protein (Fig. 1B) levels are higher in $\mathrm{BCa}$ tissues when compared with its corresponding normal tissue.

It has been reported that Sema4A could be cleaved into the extracellular compartment and functions through its receptors. ${ }^{4)}$ In this study, we also detect its expression in the serum of $\mathrm{BCa}$ patients by ELISA. The results showed that Sema4A expression was higher in the serum of $\mathrm{BCa}$ patients when compared with healthy control ones (Fig. 1C), suggesting its potential role in $\mathrm{BCa}$ progression.

Hypoxia Induces Sema4A Production in BCa Cells through a HIF-1 $\alpha$ Dependent Way Considering the elevated expression of Sema4A in BCa cells and hypoxia acts as an important contributor to $\mathrm{BCa},{ }^{12)}$ we wonder the relation between hypoxia and Sema4A. MDA-MB-231 and MCF-7 cells are chosen as the model as they are frequently used when studying the relation between hypoxia and $\mathrm{BCa} .{ }^{13-15)}$ The data showed that the expression of Sema4A in $\mathrm{BCa}$ cells could be 

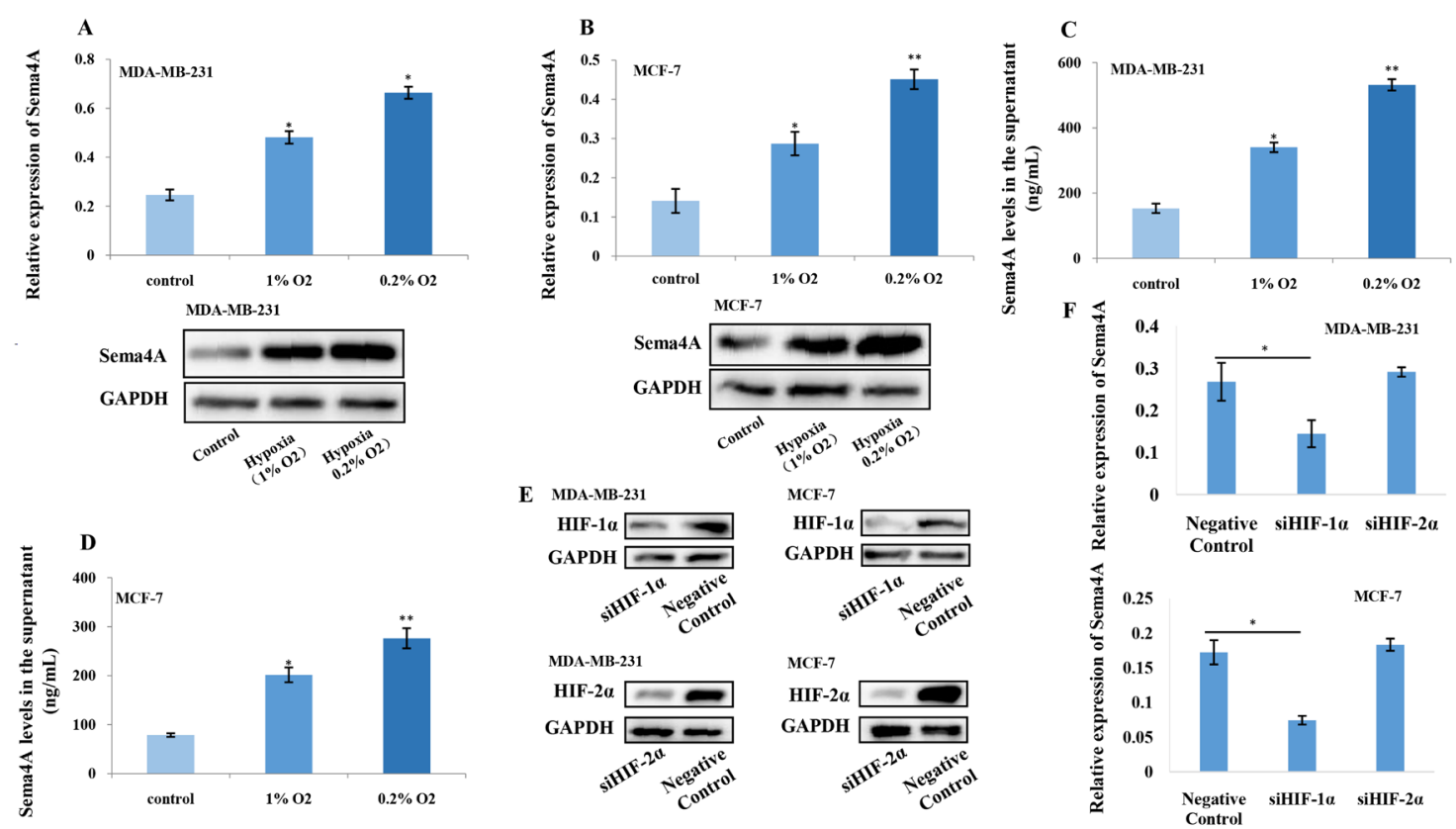

E MDA-MB-231

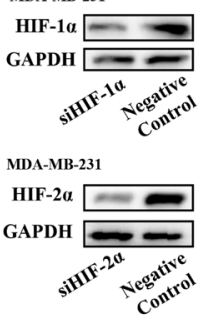

MCF-7

HIF-1 $\alpha$

GAPDH

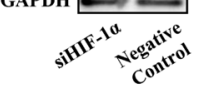

MCF-7

HIF-2o

GAPDH

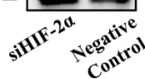
Control

G
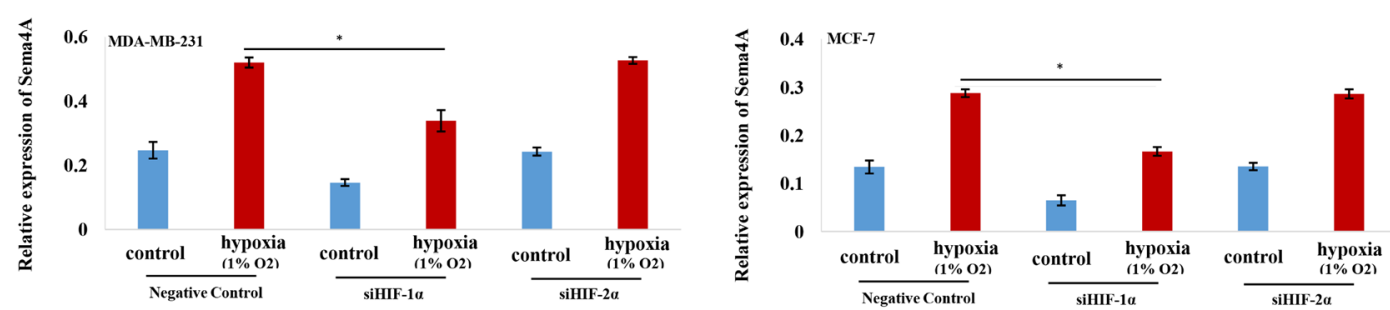

H
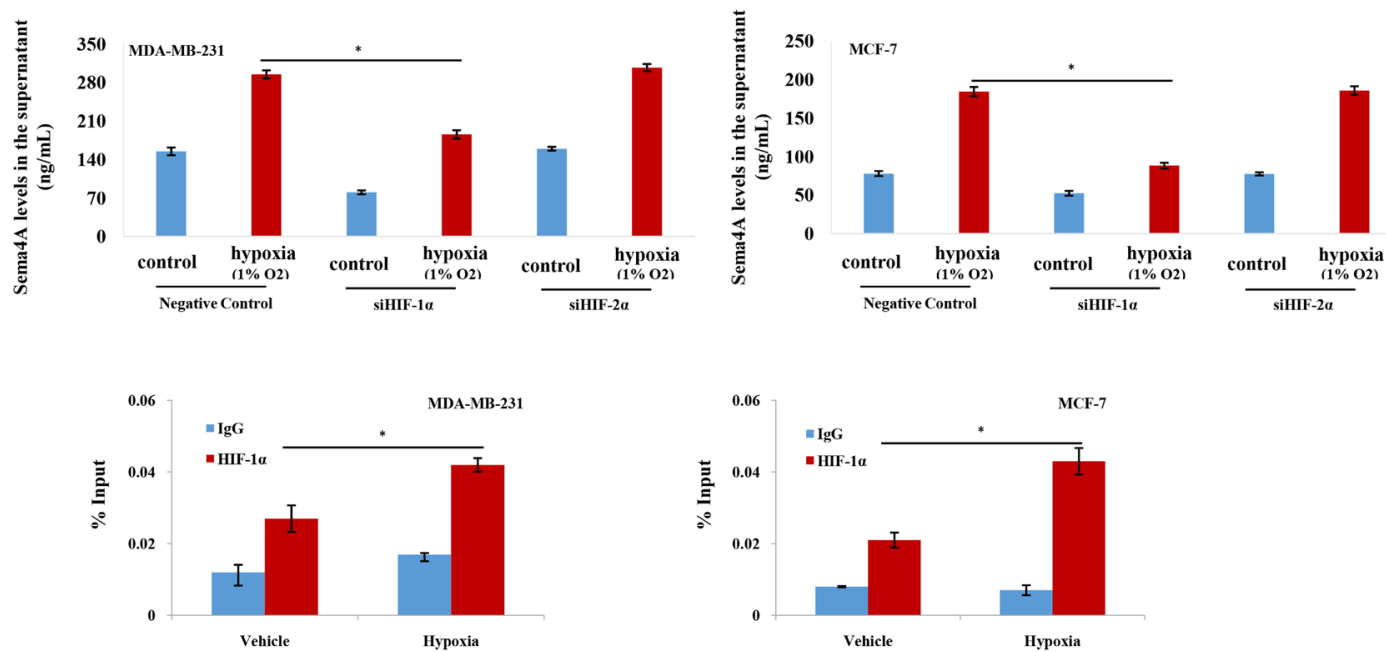

Fig. 2. Sema4A Expression Is under the Regulation of HIF-1 $\alpha$

In the presence of hypoxia ( 1 or $0.2 \% \mathrm{O}_{2}$ ), the expression of Sema4A in MDA-MB-231 (A) and MCF-7 (B) cells at RNA (upper) and protein (lower) levels were determined by RT-qPCR and Western blot. ELISA detection of the Sema4A levels in the supernatant of MDA-MB-231 (C) and MCF-7 (D) cells with the indicated hypoxic stimulation. (E) The silencing efficiency siRNA targeting HIF-1 $\alpha$ (siHIF-1 $\alpha$ ) and HIF-2 $\alpha$ (siHIF-2 $\alpha$ ) was detected in MDA-MB-231 and MCF-7 cells. (F) After transfected with siRNA targeting HIF-1 $\alpha$, HIF- $2 \alpha$ or its negative control, Sema4A mRNA expression was detected in MDA-MB-231 and MCF-7 cells. In the presence of hypoxia (1\% $\mathrm{O}_{2}$ ), the levels of Sema4a in BCa cells $(\mathrm{G})$ and their supernatant $(\mathrm{H})$ was detected by qPCR and ELISA. (I) ChIP-qPCR was performed to detect the binding of HIF-1 $\alpha$ on the promoter of Sema4A gene. Data are expressed as means \pm S.D. and compared using the unpaired $t$-test $(* p<0.05$, ** $p<0.05)$. (Color figure can be accessed in the online version.)

induced after the exposure of hypoxia ( 1 and $\left.0.2 \% \mathrm{O}_{2}\right)$ (Figs. $2 \mathrm{~A}, \mathrm{~B})$. Intriguingly, the level of Sema4A in the supernatant was also upregulated after stimulation with hypoxia (Figs. 2C, D).

As HIF- $1 \alpha$ and HIF- $2 \alpha$ are main effectors of hypoxia for tumor progression, ${ }^{12,16)}$ we subsequently analyzed the effect of HIF-1 $\alpha$ or HIF- $2 \alpha$ knocking down on the expression of Sema4A in BCa cells. The silencing efficiencies of siRNA targeting HIF-1 $\alpha$ and HIF- $2 \alpha$ were firstly detected in both cell lines (Fig. 2E). Following study showed that silencing HIF-1 $\alpha$, but not HIF-2 $\alpha$, could suppress the basal level of Sema4A in MDA-MB-231 (Fig. 2F, upper) and MCF-7 cells (Fig. 2F, lower). Of note, the induction of Sema $4 \mathrm{~A}$ in $\mathrm{BCa}$ cells in response to hypoxia $\left(1 \% \mathrm{O}_{2}\right)$ could be attenuated in the presence siRNA targeting HIF-1 $\alpha$, but not HIF-2 $\alpha$ (Fig. $2 \mathrm{G}$ ).

We also analyzed the expression of Sema4A in the super- 
natant of BCa cells. Consistent with endogenous Sema4A in $\mathrm{BCa}$ cells, the soluble Sema4A increased significantly after stimulation with hypoxia $\left(1 \% \mathrm{O}_{2}\right)$, while silencing HIF- $1 \alpha$ could antagonize this increase (Fig. 2H). Together, hypoxia is account for the increased expression of Sema4A in BCa cells.

To further validate the effect of HIF- $1 \alpha$ on Sema4A, Jaspar software was applied to predict the potential binding of HIF- $1 \alpha$ on the promoter of Sema4A gene. Next, ChIP-qPCR was performed in MDA-MB-231 and MCF-7 cells and found that HIF- $1 \alpha$ could bind to the region encompassing $-697 \mathrm{bp}-$ $-706 \mathrm{bp}$ away from its transcriptional start point of Sema4A (Fig. 2I). Importantly, the recruitment of HIF-1 $\alpha$ increased in response to hypoxic stimulation (Fig. 2I).

Sema4A Is Required for the Activity of Hypoxia towards
BCa Cells To evaluate the functional roles of Sema4A in $\mathrm{BCa}$, siRNA targeting Sema4A was designed and the one showing the most silencing efficiency was selected for following study (Fig. 3A). Next, we measured the effect of Sema4A on the activity of hypoxia. The data showed that, in response to hypoxic treatment, the production of VEGF (Figs. 3B, C) and the phosphorylated levels of MAPK, Akt and STAT3 (Figs. 3D, E) increased significantly in MDA-MB-231 and MCF-7 cells. However, silencing Sema4A could attenuate the above increase caused by hypoxic stimulation (Figs. 3B-D). We then measured the effect of recombinant human Sema4a (rhSema4a) on the expression of VEGF under hypoxic condition. More interestingly, rhSema4A in combination of hypoxic treatments induced additive increase of VEGF production (Fig.
A

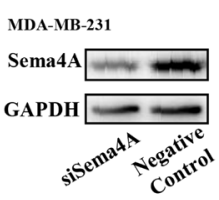

B

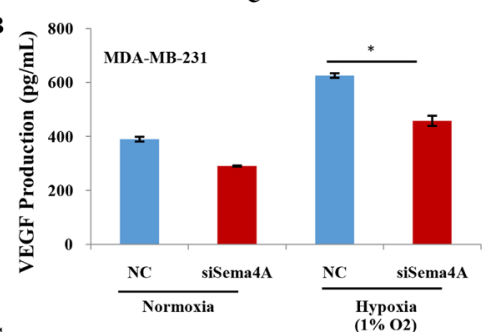

C

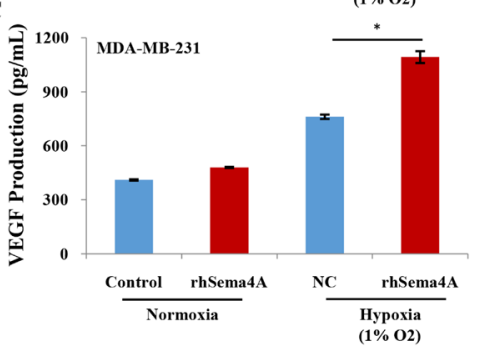

MCF-7

Sema4A wos mats

GAPDH
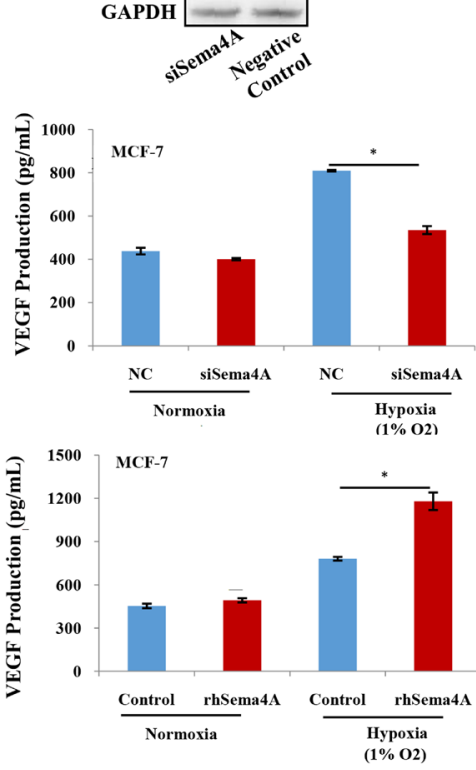

D
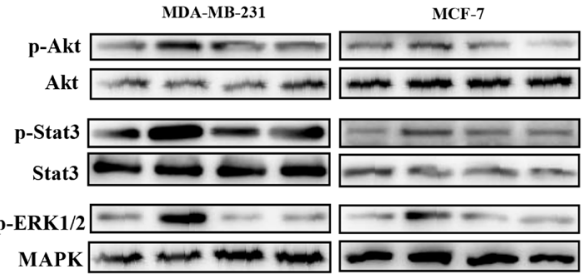

siSema4A $-\quad-\quad+\quad+\quad-\quad+\quad+\quad+$

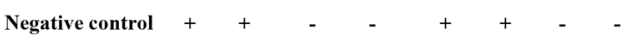

Hypoxia $(1 \%$ O2) $\quad-\quad+\quad-\quad+\quad-\quad+\quad-\quad+$

$\mathbf{E}$
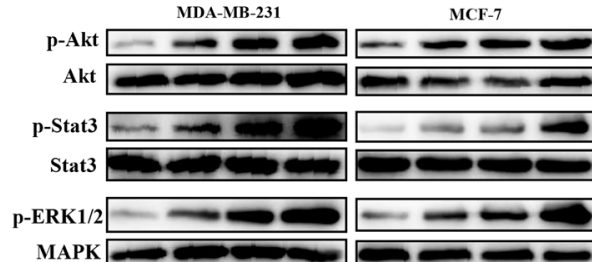

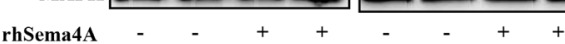

Vehicle control

Hypoxia (1\% 02)

Fig. 3. Sema4A Regulates the Activity of BCa Cells in Response to Hypoxia

(A) The silencing efficiency siRNA targeting Sema4A (siSema4A) was detected in MDA-MB-231 and MCF-7 cells. After treatment with siSema4a (B, D) or rhSema4A $(10 \mathrm{ng} / \mathrm{mL}, \mathrm{C}, \mathrm{E})$, and their respective controls, the levels of VEGF in the supernatant (B, C) and phosphorylated ERK, Akt and STAT3 (D, E) were determined in the presence of hypoxia $\left(1 \% \mathrm{O}_{2}\right)$. Data are expressed as means \pm S.D. and compared using the unpaired $t$-test $(* p<0.05)$. (Color figure can be accessed in the online version.)
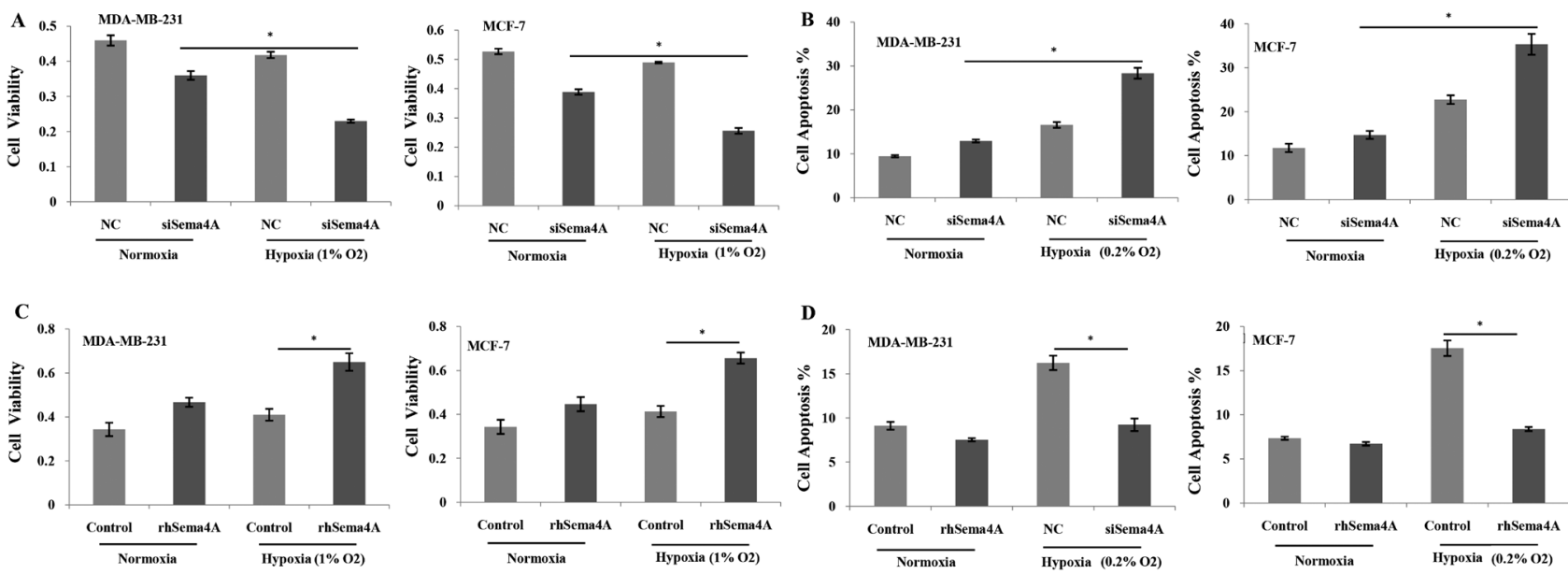

Fig. 4. Sema4A Regulates the Cell Viability and Apoptosis in BCa Cells

Under the normoxic $\left(21 \% \mathrm{O}_{2}\right)$ or hypoxic condition $\left(0.2\right.$ or $1 \% \mathrm{O}_{2}$ as indicated), the cell viability (A) and apoptosis (B) were determined by CCK8 and Annexin V. With the stimulation of rhSema4A, cell viability (C) and apoptosis (D) were detected under normoxic or hypoxic condition. Data are expressed as means \pm S.D. and compared using the unpaired $t$-test $\left({ }^{*} p<0.05\right)$. 
3C) as well as phosphorylated ERK, Akt and STAT3 when compared with hypoxia treatment alone (Fig. 3E).

Silencing Sema4A Inhibits Proliferation but Induces Apoptosis of Tumor Cells in the Presence of Hypoxia Before incubation with either normoxia or hypoxia $\left(1 \% \mathrm{O}_{2}\right)$, MCF-7 and MDA-MB-231 cells were pre-treated with siRNA targeting Sema4A or its negative control for $6 \mathrm{~h}$. Then the cell proliferation rates were then assayed for a further $24 \mathrm{~h}$. The data showed that silencing Sema4A could reduce the cell proliferation rates in both normoxia and hypoxia $\left(1 \% \mathrm{O}_{2}\right.$, Fig. 4A). More importantly, the suppression was more obvious in hypoxic condition $\left(1 \% \mathrm{O}_{2}\right.$, Fig. $\left.4 \mathrm{~A}\right)$.

Next, we measured the effects of Sema4A on cell apoptosis under normoxic or severe hypoxic condition $\left(0.2 \% \mathrm{O}_{2}\right)$ by Annexin $\mathrm{V}$. The data demonstrated that silencing Sema4A made no obvious effects on cell apoptosis in normoxia (Fig. 4B). However, following Sema4A knocking down, the apoptosis increased dramatically in the presence of hypoxia, although apoptosis could also be induced by hypoxic stimulation alone (Fig. 4B). Collectively, these data indicates that silencing Sema4A may inhibit the progression of BCa.

rhSema4A Protects BCa Cells against Apoptosis Induced by Severe Hypoxia To investigate that function of Sema4A in its soluble form, rhSema4A was applied to detect its effects on the biological activity of BCa cells. The results showed that treatment with rhSema4A could up-regulate the cell proliferation of $\mathrm{BCa}$ cells, which could be further enhanced with the stimulation of hypoxia $\left(1 \% \mathrm{O}_{2}\right)$ (Fig. 4C). We also applied severe hypoxia $\left(0.2 \% \mathrm{O}_{2}\right)$ to induce more cell apoptosis and determine the effect of rhSema4A on it. The results showed that rhSema4A treatment could provide resistance to hypoxiainduced cell apoptosis (Fig. 4D).

\section{DISCUSSION}

Recent study demonstrated the intense relation between Sema4A and tumor. ${ }^{4,10,11)}$ It was shown that germline variants in the Sema4A gene could predispose to familial colorectal cancer type $\mathrm{X}^{17)}$ Sema4A could also promote the drug resistance in hepatocellular carcinoma. ${ }^{11)}$ In $\mathrm{BCa}$, Sema4A could regulate the migration of $\mathrm{BCa}$ cells through interacting with its receptor. ${ }^{11)}$ However, the exact roles and its pathological mechanism in $\mathrm{BCa}$ remain unknown. In this study, our data revealed that Sema4A may promote the progression of $\mathrm{BCa}$ based on the following facts. Firstly, Sema4A expression is more abundant in $\mathrm{BCa}$ tissues than its adjacent non-cancerous ones. In addition, the serum levels of Sema4A are also higher in $\mathrm{BCa}$ patients than normal controls. Secondly, silencing Sema4A could inhibit the cell proliferation, but induce apoptosis of BCa cells. Tumor formation assay in vivo would be needed to further support its oncogenic role of Sema4A in $\mathrm{BCa}$.

As the highly proliferative tumor cells require more oxygen consumption, hypoxia exits in the tumor microenvironment. ${ }^{18)}$ Recently, it has been increasingly recognized that the tumor microenvironment is closely related with tumor progression and HIF-1 $\alpha$ acts as the main regulator to adapt to intratumoral hypoxia. ${ }^{18,19)}$ Another finding in this study is the cross-talk between Sema4A and hypoxic activity. Our data showed that Sema4A expression is directly regulated by HIF-1 $\alpha$ in the presence of hypoxia. Furthermore, Sema4A may act as an ef- fector of $\mathrm{BCa}$ cells in response to hypoxia as demonstrated by the facts that the effects of Sema4A knocking-down on cell proliferation and apoptosis was more obvious in the presence of hypoxia. Besides, in response to hypoxia, the most important characteristics of $\mathrm{BCa}$ cells are the abnormal production of $\mathrm{VEGF}^{20)}$ and the deregulated activation of Akt, MAPK and STAT3 signaling. ${ }^{21-23)}$ Therefore, reduction of the above signaling may offer benefits in BCa therapeutic efficacy. Our results demonstrated that silencing Sema4A could effectively attenuate the production of VEGF, as well as the activation of Akt, MAPK and STAT3 with the stimulation of hypoxia, indicating its potential for treatment target. As administration of Sema4A could diminish the efficacy of interferon- $\beta$ (IFN- $\beta$ ) in experimental autoimmune encephalomyelitis, ${ }^{24)}$ further study would also be needed to study the effects of Sema4A on chemo-sensitiveness of $\mathrm{BCa}$ cells.

High serum Sema4a levels were observed in multiple sclerosis (MS) and rheumatoid arthritis patients. ${ }^{9,25)}$ Besides, Sema4A in its soluble form could also regulate the biological activity of synovial fibroblasts of rheumatoid arthritis (RASFs) as well. ${ }^{9)}$ In this study, we also found that the secretion of Sema4A increased with the stimulation of hypoxia and soluble Sema4A, in turn, could regulate the biological activity of $\mathrm{BCa}$ cells, supporting its pathological activity in the microenvironment during tumor progression. Previous study demonstrated that Sema4A could enhance lung fibrosis via Plexin D1 receptor, ${ }^{26)}$ but is required for optimal activation of mammalian target of rapamycin (mTOR)C1 in $\mathrm{CD} 8(+) \mathrm{T}$ cells through Plexin B2 receptor. ${ }^{27)}$ These data suggest that Sema4A's functions differ from cell types and receptors and the receptor of Sema4A involving BCa needs to be clarified.

Taken together, these results indicate that hypoxic induction of Sema4A contributes to the abnormal proliferation and apoptosis of BCa cells. Specific targeting Sema4A may be a novel treatment avenue for $\mathrm{BCa}$.

Acknowledgments This work was supported by the National Natural Science Foundation of China (Grant Nos. 81572544, 81772760, 81670046), Key Research and Development Project of Shandong (Grant No. 2016GSF201166), Scientific research fund of Jinan University (XKY1522), Supporting Fund for Teacher's research of Jining Medical University (JY2017FS001), Shandong Taishan Scholarship (Grant No. tsqn20161076) and Innovation Project of Shandong Academy of Medical Sciences.

Conflict of Interest The authors declare no conflict of interest.

\section{REFERENCES}

1) Alto LT, Terman JR. Semaphorins and their signaling mechanisms. Methods Mol. Biol., 1493, 1-25 (2017).

2) Nishide M, Kumanogoh A. The role of semaphorins in immune responses and autoimmune rheumatic diseases. Nat. Rev. Rheumatol., 14, 19-31 (2018).

3) Neufeld G, Mumblat Y, Smolkin T, Toledano S, Nir-Zvi I, Ziv K, Kessler O. The role of the semaphorins in cancer. Cell Adh. Migr., 10, 652-674 (2016)

4) Ito D, Kumanogoh $\mathrm{A}$. The role of Sema4A in angiogenesis, immune responses, carcinogenesis, and retinal systems. Cell Adh. Migr., 10, 692-699 (2016). 
5) Lynch JP, Werder RB, Loh Z, Sikder MAA, Curren B, Zhang V, Rogers MJ, Lane K, Simpson J, Mazzone SB, Spann K, Hayball J, Diener K, Everard ML, Blyth CC, Forstner C, Dennis PG, Murtaza N, Morrison M, O Cuív P, Zhang P, Haque A, Hill GR, Sly PD, Upham JW, Phipps S. Plasmacytoid dendritic cells protect from viral bronchiolitis and asthma through semaphorin 4a-mediated $\mathrm{T}$ reg expansion. J. Exp. Med., 215, 537-557 (2018).

6) Abid A, Ismail M, Mehdi SQ, Khaliq S. Identification of novel mutations in the SEMA4A gene associated with retinal degenerative diseases. J. Med. Genet., 43, 378-381 (2006).

7) Kumanogoh A, Marukawa S, Suzuki K, Takegahara N, Watanabe C, Ch'ng E, Ishida I, Fujimura H, Sakoda S, Yoshida K, Kikutani H. Class IV semaphorin Sema4A enhances T-cell activation and interacts with Tim-2. Nature, 419, 629-633 (2002).

8) Meda C, Molla F, De Pizzol M, Regano D, Maione F, Capano S, Locati M, Mantovani A, Latini R, Bussolino F, Giraudo E. Semaphorin 4A exerts a proangiogenic effect by enhancing vascular endothelial growth factor-A expression in macrophages. J. Immunol., 188, 4081-4092 (2012).

9) Wang L, Song G, Zheng Y, Tan W, Pan J, Zhao Y, Chang X. Expression of Semaphorin 4A and its potential role in rheumatoid arthritis. Arthritis Res. Ther., 17, 227 (2015).

10) Pan JX, Wang F, Ye LY. Doxorubicin-induced epithelial-mesenchymal transition through SEMA 4A in hepatocellular carcinoma. Biochem. Biophys. Res. Commun., 479, 610-614 (2016).

11) Sun T, Yang L, Kaur H, Pestel J, Looso M, Nolte H, Krasel C, Heil D, Krishnan RK, Santoni MJ, Borg JP, Bünemann M, Offermanns S, Swiercz JM, Worzfeld T. A reverse signaling pathway downstream of Sema4A controls cell migration via Scrib. J. Cell Biol., 216, 199-215 (2017).

12) Wang L, Fan J, Yan CY, Ling R, Yun J. Activation of hypoxiainducible factor- $1 \alpha$ by prolonged in vivo hyperinsulinemia treatment potentiates cancerous progression in estrogen receptor-positive breast cancer cells. Biochem. Biophys. Res. Commun., 491, 545-551 (2017).

13) Rezaeian AH, Li CF, Wu CY, Zhang $X$, Delacerda J, You MJ, Han F, Cai Z, Jeong YS, Jin G, Phan L, Chou PC, Lee MH, Hung MC, Sarbassov D, Lin HK. Ahypoxia-responsive TRAF6-ATM-H2AX signalling axis promotes HIF1 $\alpha$ activation, tumorigenesis and metastasis. Nat. Cell Biol., 19, 38-51 (2017).

14) Zhang C, Samanta D, Lu H, Bullen JW, Zhang H, Chen I, He X, Semenza GL. Hypoxia induces the breast cancer stem cell phenotype by HIF-dependent and ALKBH5-mediated $\mathrm{m}^{6} \mathrm{~A}$-demethylation of NANOG mRNA. Proc. Natl. Acad. Sci. U.S.A., 113, E2047E2056 (2016).

15) Samanta D, Gilkes DM, Chaturvedi P, Xiang L, Semenza GL. Hypoxia-inducible factors are required for chemotherapy resistance of breast cancer stem cells. Proc. Natl. Acad. Sci. U.S.A., 111, E5429E5438 (2014).

16) Hong SE, Jin HO, Kim HA, Seong MK, Kim EK, Ye SK, Choe TB, Lee JK, Kim JI, Park IC, Noh WC. Targeting HIF-1 $\alpha$ is a prereq- uisite for cell sensitivity to dichloroacetate (DCA) and metformin. Biochem. Biophys. Res. Commun., 469, 164-170 (2016).

17) Schulz E, Klampfl P, Holzapfel S, Janecke AR, Ulz P, Renner W, Kashofer K, Nojima S, Leitner A, Zebisch A, Wölfler A, Hofer S, Gerger A, Lax S, Beham-Schmid C, Steinke V, Heitzer E, Geigl JB, Windpassinger C, Hoefler G, Speicher MR, Boland CR, Kumanogoh A, Sill H. Germline variants in the SEMA4A gene predispose to familial colorectal cancer type X. Nat. Commun., 5, 5191 (2014).

18) Tang ZN, Zhang F, Tang P, Qi XW, Jiang J. Hypoxia induces RANK and RANKL expression by activating HIF-1 $\alpha$ in breast cancer cells. Biochem. Biophys. Res. Commun., 408, 411-416 (2011).

19) Karlenius TC, Shah F, Di Trapani G, Clarke FM, Tonissen KF. Cycling hypoxia up-regulates thioredoxin levels in human MDAMB-231 breast cancer cells. Biochem. Biophys. Res. Commun., 419, 350-355 (2012).

20) Okada K, Osaki M, Araki K, Ishiguro K, Ito H, Ohgi S. Expression of hypoxia-inducible factor (HIF-1alpha), VEGF-C and VEGF-D in non-invasive and invasive breast ductal carcinomas. Anticancer Res., 25, 3003-3009 (2005).

21) Wu MZ, Chen SF, Nieh S, Benner C, Ger LP, Jan CI, Ma L, Chen $\mathrm{CH}$, Hishida T, Chang HT, Lin YS, Montserrat N, Gascon P, Sancho-Martinez I, Izpisua Belmonte JC. Hypoxia drives breast tumor malignancy through a TET-TNF $\alpha$-p38-MAPK signaling axis. Cancer Res., 75, 3912-3924 (2015).

22) Leszczynska KB, Foskolou IP, Abraham AG, Anbalagan S, Tellier C, Haider S, Span PN, O’Neill EE, Buffa FM, Hammond EM. Hypoxia-induced p53 modulates both apoptosis and radiosensitivity via AKT. J. Clin. Invest., 125, 2385-2398 (2015).

23) Seo HS, Ku JM, Choi HS, Woo JK, Jang BH, Shin YC, Ko SG Induction of caspase-dependent apoptosis by apigenin by inhibiting STAT3 signaling in HER2-overexpressing MDA-MB-453 breast cancer cells. Anticancer Res., 34, 2869-2882 (2014).

24) Koda T, Okuno T, Takata K, Honorat JA, Kinoshita M, Tada S, Moriya M, Sakoda S, Mochizuki H, Kumanogoh A, Nakatsuji Y. Sema4A inhibits the therapeutic effect of IFN- $\beta$ in EAE. J. Neuroimmunol., 268, 43-49 (2014).

25) Koda T, Namba A, Nakatsuji Y, Niino M, Miyazaki Y, Sugimoto T, Kinoshita M, Takata K, Yamashita K, Shimizu M, Fukazawa T, Kumanogoh A, Mochizuki H, Okuno T. Beneficial effects of fingolimod in MS patients with high serum Sema4A levels. PLOS ONE, 13, e0193986 (2018).

26) Peng HY, Gao W, Chong FR, Liu HY, Zhang JI. Semaphorin 4A enhances lung fibrosis through activation of Akt via PlexinD1 receptor. J. Biosci., 40, 855-862 (2015).

27) Ito $\mathrm{D}$, Nojima $\mathrm{S}$, Nishide $\mathrm{M}$, Okuno $\mathrm{T}$, Takamatsu $\mathrm{H}$, Kang $\mathrm{S}$, Kimura T, Yoshida Y, Morimoto K, Maeda Y, Hosokawa T, Toyofuku T, Ohshima J, Kamimura D, Yamamoto M, Murakami M, Morii E, Rakugi H, Isaka Y, Kumanogoh A. mTOR Complex signaling through the SEMA4A-plexin B2 axis is required for optimal activation and differentiation of CD8+ T Cells. J. Immunol., 195, 934-943 (2015). 